\title{
Assessment of empirical antibiotic therapy in a tertiary-care hospital: An observational descriptive study
}

\author{
Antony $\mathrm{GK}(*)$, Manjaly $\mathrm{JP}(* *)$, Mukhtar L(***) and Alva JP(****). \\ *Resident, Dept. Of Medicine, Father Muller Medical College, Mangaluru. \\ **Senior Resident, Dept.of Medicine, Father Muller Medical College, Mangaluru. \\ ***Intern, Father Muller Medical College, Mangaluru. \\ ****Dean and Professor of Medicine, Father Muller Medical College, Mangaluru.
}

\begin{abstract}
Background: Empirical antibiotics when employed in clinical practice should be supported with culture results or valid clinical signs/symptoms. Modification of the empirical regimen is recommended when warranted in order to control antimicrobial overuse and resistance. This study aimed to assess the frequency of starting empirical intravenous antibiotics, the characteristics of this empirical regimen and the clinical characteristics of the patients at the time of start of therapy. It also sought to determine the change in regimen within the first 5 days of admission based on clinical profile and culture reports.

Methods: This is a descriptive study enrolling 100 subjects who were administered antibiotics on admission, with a hospital stay for a minimum of 5 days. They were assessed for common symptoms viz. fever, cough etc. Investigative modalities sent for were charted, with special importance to leucocyte counts, chest radiographs and culture tests. Addition of a second antibiotic if any was documented and the reason for the same was reviewed.

Findings: Of all patients initiated on antibiotics, fever was the predominant symptom (64\%) followed by GI symptoms (39\%). Twenty percent had tachycardia documented on clinical examination. Respiratory tract infections accounted for majority of admissions included in the study (35\%). Among patients initiated on antibiotics, only $49 \%$ had elevated leucocyte counts. Majority of antibiotics initiated belonged to Rank 2(69\%). Of all the patients, thirty one percent had their blood cultures sent, while seven and sixteen percent respectively had urine and sputum cultures dispatched. Thirty seven percent had chest radiographs with findings while nineteen percent had documented pyuria on urine examination. Twenty nine percent had a second antibiotic added. Worsening general condition was the most frequent reason for the change followed by change as per culture positivity.

Interpretation: Broad spectrum empirical antibiotic therapy is common, even when clinical signs and symptoms pertaining to an underlying infection are absent. Relevant imaging and collection of cultures at the time of initiation of antibiotics is essential to guide the continuation of the drug. Hence the importance of stewardship programs to oversee mechanisms for optimal prescribing of broad spectrum antibiotics.

All correspondence to Dr. George K Antony,Resident, Dept. of Medicine. Father Muller Medical College, Mangaluru.
\end{abstract}

\section{Introduction}

Multi-drug resistant bacteria are posing a major threat to modern health care ${ }^{(1)}$ Overprescribing of antimicrobials are a major factor leading to drug resistance. ${ }^{(2)}$ Broad spectrum, higher antibiotics are increasingly being administered in today's practice especially in tertiary care facilities with a prolonged duration of use. ${ }^{(3,4)}$ Hence more effective implementation of antimicrobial stewardship in the inpatient setting is warranted. . $^{(3,5)}$

Such monitoring programs aim to minimize unnecessary or inappropriate antibiotic therapy, mainly by the restriction or audit and review of antimicrobial prescriptions. A common strategy adopted is what is referred to as therapy optimization. This involves initiation of empirical antibiotic cover without delay which is then followed by discontinuation or streamlining to a regimen with the narrowest possible spectrumbased on relevant diagnostic information ${ }^{(6)}$ The first reassessment should be carried out as soon as culture data is made available, and removal of one or more antibiotics is made feasible by clinical improvement and bacteriological reports. ${ }^{(6,7)}$

Very few studies actually delve into how often and what clinical factors guide the optimization of empirical antibiotic use in the general hospital population. Identifying the factors associated with the initiation and cessation, and change of modifiable empirical therapy is the key to reducing unnecessary antibiotic exposure and hence minimize the development of resistance and adverse outcomes. ${ }^{(8,9)}$ 


\section{Aim of the study}

To assess the frequency of starting empirical intravenous antibiotics, the characteristics of this empirical regimen and the clinical characteristics of the patients at the time of start of therapy. It also sought to determine the change in regimen within the first 5 days of admission based on clinical profile and culture reports.

\section{Materials And Methods}

Study design: This is a descriptive study enrolling 100 patients admitted to Father Muller Medical College, Mangalore meeting the selection criteria.The study spanned three months from April 2016 and included patients admitted to Father Muller Medical College, with a hospital stay fora minimum of 5 days and initiated on antibiotics on the day of admission. Immunocompromised individuals and those on long term steroidswere excluded. The 100 study subjects enrolled were evaluated for comorbidities, rank of antibiotic initiated on day 1 , the frequency and route of administration. They were assessed for symptoms of fever(grade and duration), cough, gastrointestinal symptoms and dysuria. Clinical examination was carried out and special attention was given to signs viz. tachycardia/bradycardia, and hypotension. The provisional diagnosis was made note of. The investigations sent for were reviewed which included leucocyte count, differential counts, urine examination findings, chest radiograph findings and culture samples (blood, urine and sputum) and appropriate scoring was given. Addition of a second antibiotic if any was documented. The day and indication for the same was reviewed and the appropriate rank of the antibiotic(see below) was charted.

\begin{tabular}{|l|l|}
\hline RANK & ANTIBIOTICS \\
\hline 1 & First generation cephalosporin Other narrow spectrum \\
\hline 2 & $\begin{array}{l}\text { Fluroquinolones, third-generation cephalosporins, fluroquinolones } \\
\text { plus metronidazole, third generation cephalosporins plus macrolide, } \\
\text { other broad spectrum }\end{array}$ \\
\hline 3 & $\begin{array}{l}\text { Anti-pseudomonalpencillin plus vancomycin, anti- } \\
\text { pseudomonalpencillin, vancomycin, anti-pseudomonalpencillin plus } \\
\text { fluroquinolones and other extended spectrum. }\end{array}$ \\
\hline 4 & Carbapenam and other restricted drugs. \\
\hline
\end{tabular}

\section{Results and observations}

Figure1: Incidence of signs and symptoms in study subjects

INCIDENCE OF SIGNS AND SYMPTOMS

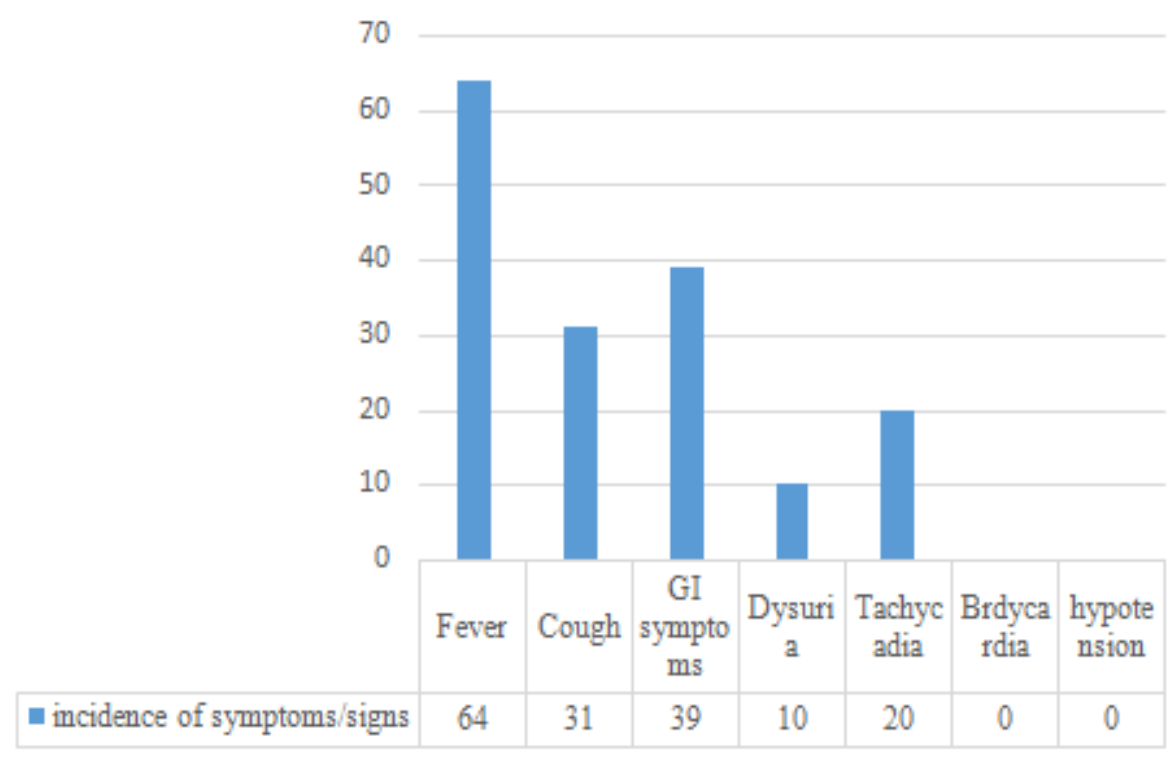


Figure 2: Various provisional diagnosis at admission in study subjects PROVISIONAI DIAONOSIS AT ADMISSION

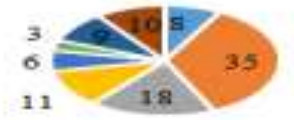

- BLOOD STRENMITNFECTIONS

- RESPIRATORX TRACT MNPECTIONS

- URTNARYTRACT INTECTIONS

- CELLULTIS

- DENGUE

DINIARIA

- FERETLE TL L NE38 UNGP

Figure 3: Rank of antibiotic and percentage of use

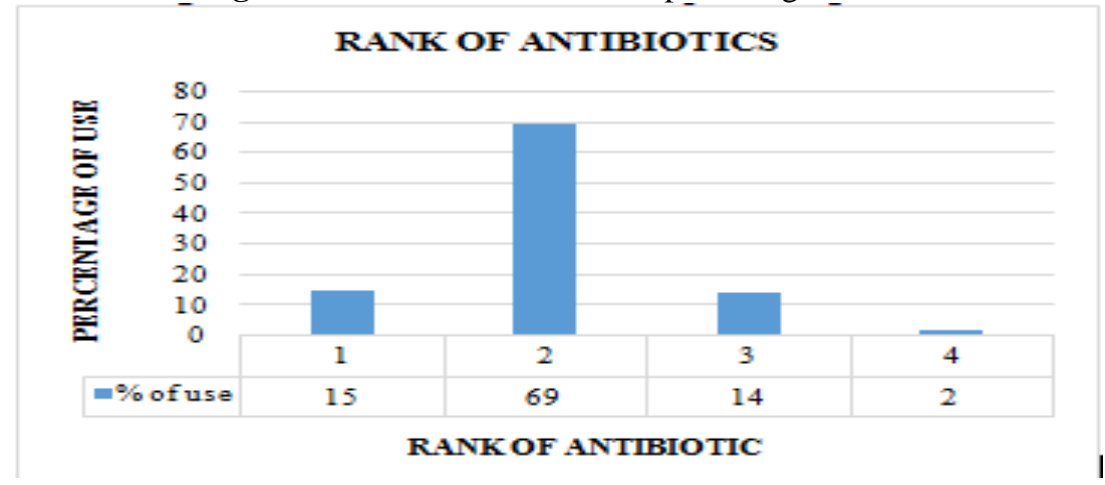

Figure 4: Daily frequency of administration of antibiotic
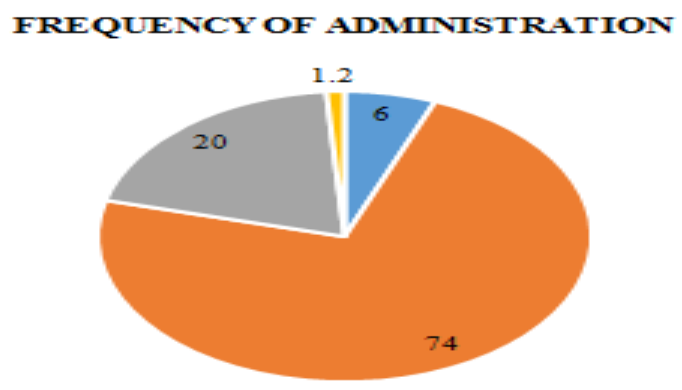

$=O D=B I D=T I D=$ | $=$ =ID

Figure 5: Route of administration of various antibiotics

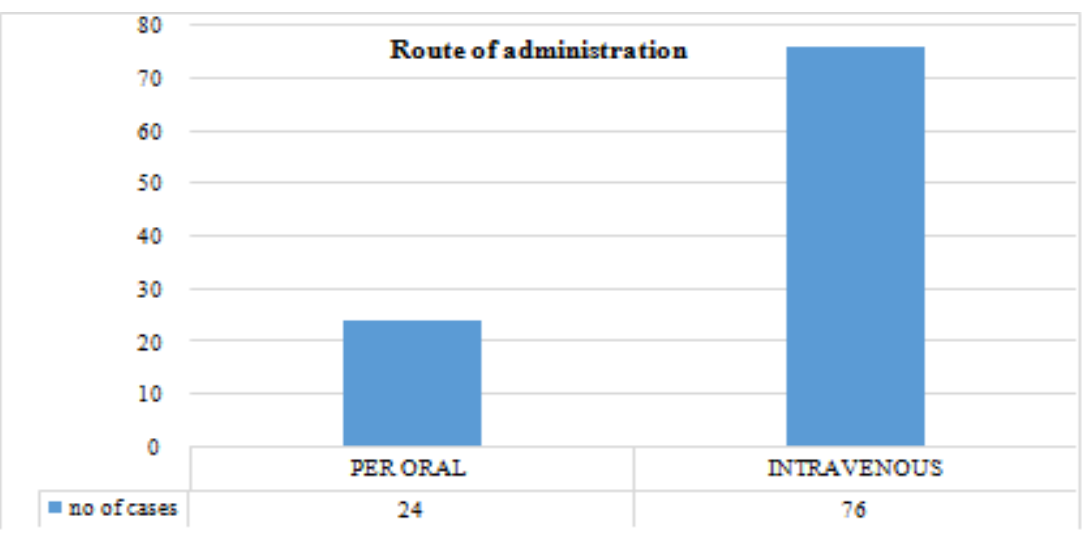

Figure 6: Investigative modalities sent/with findings at admission 


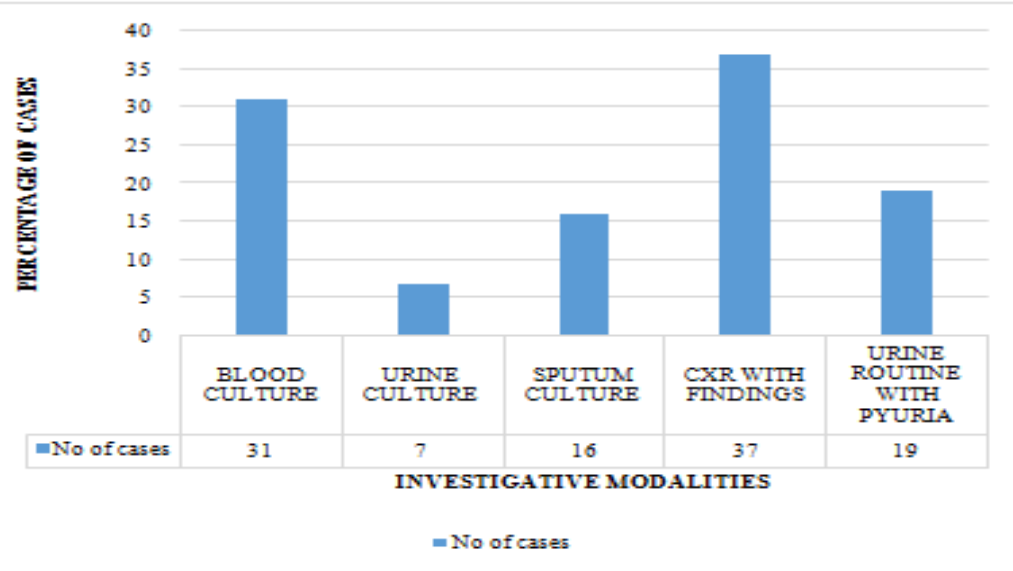

Figure 7: Addition of a second antibiotic ADDITION OF SECOND ANTIBIOTIC

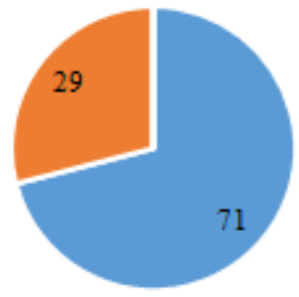

Figure 8: Indications for addition of a second antibiotic

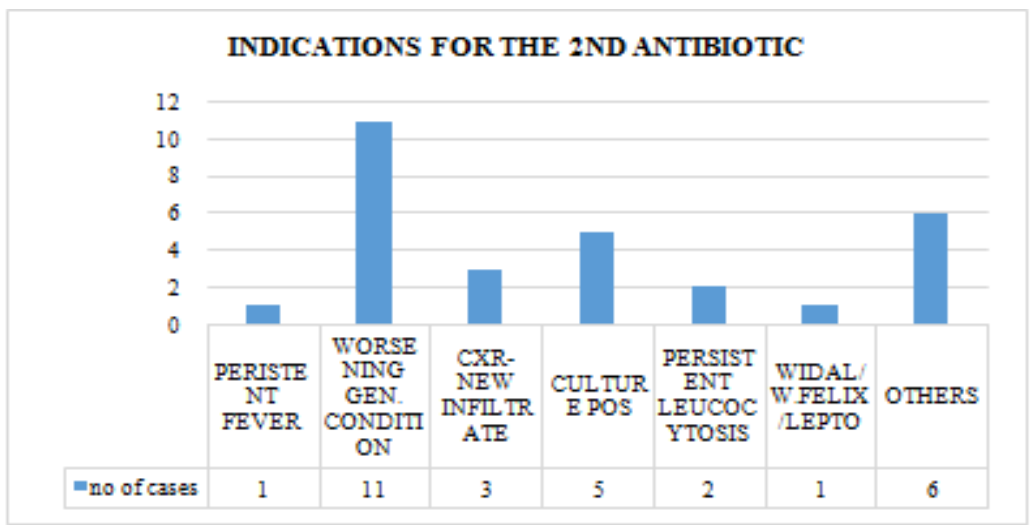

Figure 9: Day of initiation of the second antibiotic

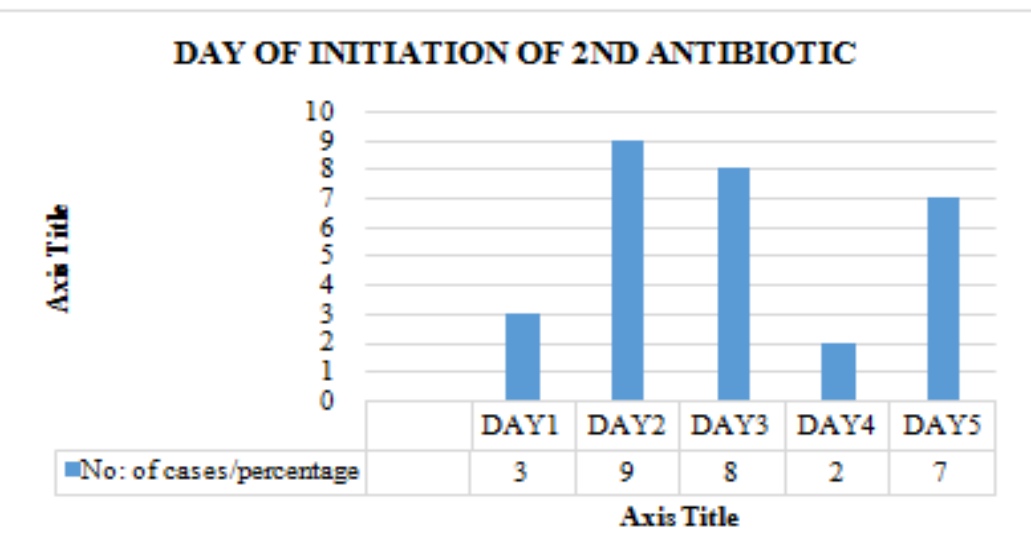

Figure 10: Rank of the second antibiotic added 
THE SECOND ANTIBIOTIC ADDED

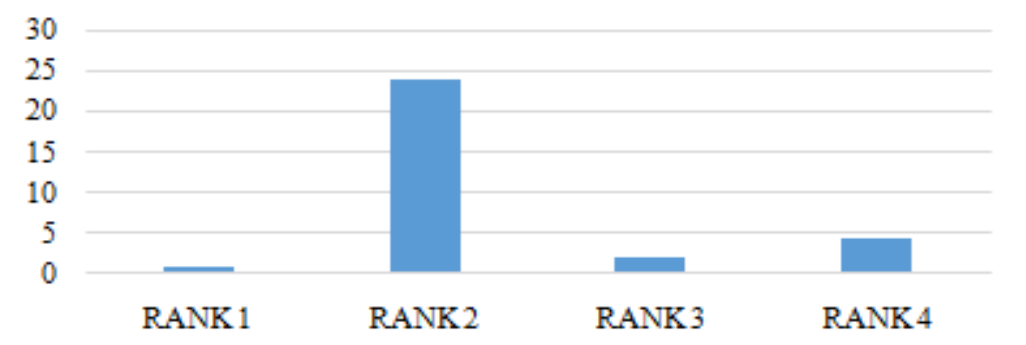

Figure 11: Rank of antibiotic with respect to leucocyte count



Figure 12: Rank of antibiotic with respect to blood cultures sent/not sent

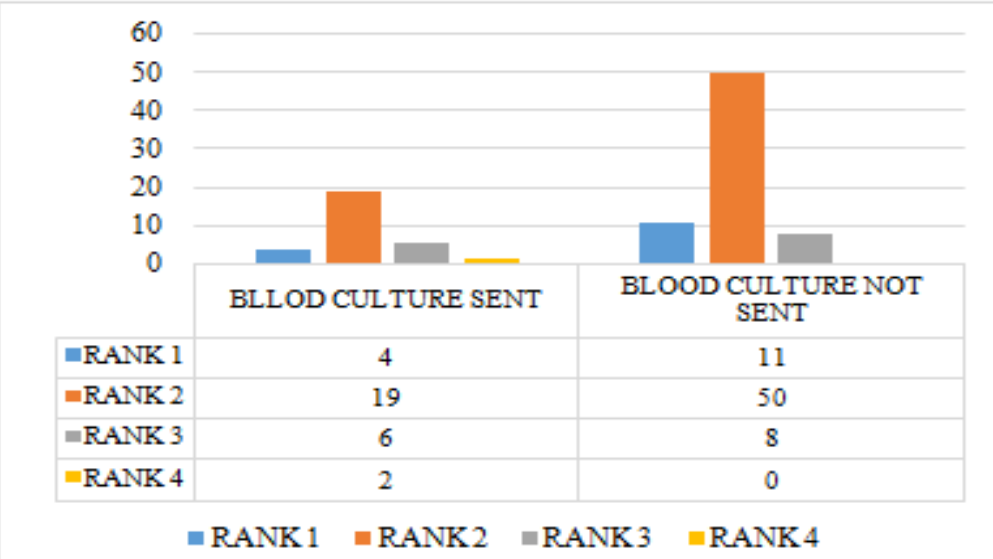

Figure 13: Rank of antibiotic with respect to urine cultures sent/not sent

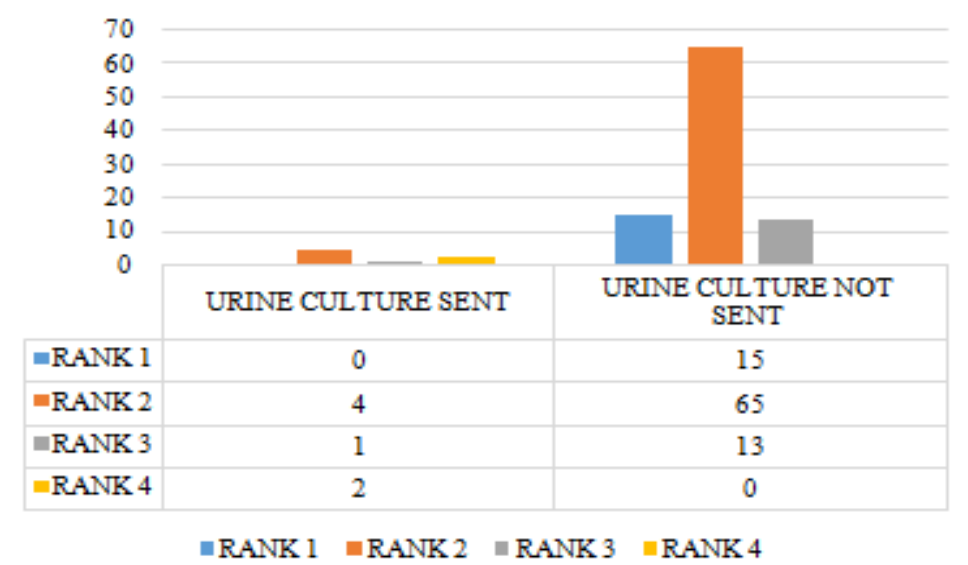

Figure 14 Rank of antibiotics with respect to sputum cultures sent/not sent 




Figure 15: Rank of antibiotic with respect to presence/absence of fever

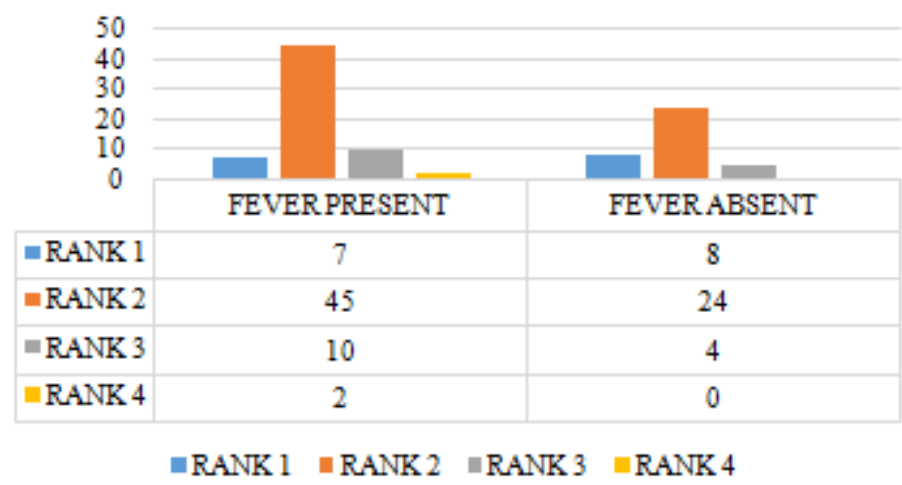

Of all patients initiated on antibiotics, fever was the predominant symptom $(64 \%)$ followed by GI symptoms (39\%). Cough was present in $31 \%$. Ten percent had dysuria on presentation. Twenty percent had tachycardia documented on clinical examination. (fig.1). Respiratory tract infections accounted for majority of admissions included in the study(35\%). Urinary tract infections stood second(18\%) followed by soft tissue infections, gastrointestinal infections and febrile illnesses. (fig.2)

Among patients initiated on antibiotics, $49 \%$ had normal leucocyte counts. An equal number had increased counts whereas 2 patients had decreased leucocyte counts.

Of the antibiotics initiated, majority belonged to Rank 2(69\%) followed by Rank 1 and Rank 3(fig.3). The route of administration was predominantly intravenous (76\%). Twice a day dosages predominated over others (fig.4). Of all the patients, thirty one percent had their blood cultures sent, while seven and sixteen percent respectively had urine and sputum cultures dispatched. Thirty seven percent had chest radiographs with findings while nineteen percent had documented pyuria on urine examination.

A change in antibiotic was also documented. Twenty nine percent had a second antibiotic added(fig.7). Worsening general condition was the most frequent reason for the change followed by change as per culture positivity (fig.8). The second antibiotic was added mostly on the $2^{\text {nd }}$ and $3^{\text {rd }}$ day of admission. Rank 2 antibiotics were the most frequently added followed by Rank 4 antibiotics.

\section{Discussion}

This study clearly shows that around a third of patients started on antibiotics did not have fever. This is well supported by studies by Braykov et al who conducted a similar study across six centers in the United States, and came to a conclusion that broad spectrum antibiotic therapy was common even when clinical signs of infection were absent. ${ }^{(10)}$ They also observed that at the time of starting antibiotics, about half of the ordered radiology and microbiology results did not identify an infection. In this study only a third of the patients had radiographic findings warranting antibiotic use. This again is similar to findings by other studies that upto half of antimicrobials started in hospitals are unnecessary. ${ }^{(11,12)}$

\section{Conclusion}

The present study shows that a worsening of clinical condition had greater implications in changing antibiotics compared to culture positivity and others. Relevant imaging and collection of cultures at the time of initiation of antibiotics is essential. The need for a thorough clinical examination in modern practice and also the 
importance of clean and protocol guided, appropriate collection techniques of specimen harvest for culture and sensitivity testing have been highlighted by this study.

Through this study we stress the need for improving the current scenario of inpatient antibiotic use. More attention should be given to patients started on antimicrobials in the absence of common indicators viz. fever and abnormal counts. Suspected respiratory, GI and urinary tract infections where imaging and cultures return negative and suggest the absence of infection preclude the use of antimicrobials. Hence the importance of stewardship programs to oversee mechanisms for optimal prescription of broad spectrum antibiotics. This calls for all health care givers to be adequately trained in the appropriate use of antimicrobials.

\section{References}

[1]. Cosgrove SE. The relationship between antimicrobial resistance and patient outcomes: mortality, length of hospital stay, and health care costs. Clin Infect Dis 2006; 42(suppl 2): S82-89.

[2]. Goossens $\mathrm{H}$ et al. Outpatient antibiotic use in Europe and association with resistance: a cross national database study. Lancet 2005; 365:579-87.

[3]. Fridkin S, Baggs J, Fagan R et al. Vital signs: improving antibiotic use among hospitalized patients. MMWR Morb Mortal Wkly Rep 2014; 63: 194-200.

[4]. Werner NL, Hecker MT, Sethi AK, Donskey CJ. Unnecessary use of fluroquinolone antibiotics in hospitalized patients. BMC Infect Dis. 2011; 11:187.

[5]. Centers for disease control and prevention. CDC's campaign to prevent antimicrobial resistance in health-care settings. MMWR Morb Mortal Wkly Rep. 2002; 51:343.

[6]. Masterton RG. Antibiotic de-escalation. Crit Care Clin 2011; 27:149-62.

[7]. Alvarez-Lerma F, Alvarez B, Luque P et al. Empiric broad-spectrum antibiotic therapy of nosocomial pneumonia in the intensive care unit: a prospective observational study. Crit Care 2006; 10:R78.

[8]. Shehab N, Patel PR, Srinivasan A, Budnitz DS. Emergency department visits for antibiotic-associated adverse events. Clin Infect Dis. 2008; 47:735-43.

[9]. OlofssonSk, Cars O. Optimizing drug exposure to maintain selection of antibiotic resistance. Clin Infect Dis 2007; 45(suppl 2):S129-36.

[10]. Braykov NP, Morgan DJ, Marin LS et al. Assessment of empirical antibiotic therapy optimization in six hospitals: an observational cohort study. Lancet Infect Dis 2014:1220-27.

[11]. Cuisini A, Rampini SK, Bansal V. et al. Different patterns of inappropriate antimicrobial use in surgical and medical units at a tertiary care hospital in Switzerland: a prevalence survey. PloS One 2010; 5:e14011.

[12]. Hecker MT, Aron DC, Patel NP et al. Unnecessary use of antimicrobials in hospitalized patients: current patterns of misuse with an emphasis on the antianaerobic spectrum of activity. Arch Intern Med 2003; 163: 972-78 